\title{
Vitamin K deficiency bleeding in Australian infants 1993-2017: an Australian Paediatric Surveillance Unit study
}

\author{
Yvonne Zurynski, ${ }^{1,2}$ Cameron J Grover (1) , ${ }^{3}$ Bin Jalaludin, ${ }^{4,5}$ Elizabeth J Elliott ${ }^{1,6,7}$
}

For numbered affiliations see end of article.

\section{Correspondence to} Professor Elizabeth J Elliott, Faculty of Medicine and Health, Discipline of Child and Adolescent Health, University of Sydney, Sydney, New South Wales 2006, Australia; elizabeth.elliott@health.nsw. gov.au

YZ and CJG are joint first authors.

Received 28 October 2018 Revised 13 August 2019 Accepted 19 August 2019 Published Online First 13 September 2019

\section{SLinked}

- https://doi.org/10.1136/ archdischild-2019-318117

Check for updates

(C) Author(s) (or their employer(s)) 2020. No commercial re-use. See rights and permissions. Published by BMJ.

To cite: Zurynski Y, Grover CJ Jalaludin B, et al.

Arch Dis Child

2020;105:433-438.

\section{ABSTRACT}

Objective To undertake surveillance of vitamin $\mathrm{K}$ deficiency bleeding (VKDB) in Australia from 1993 to 2017, during a time of change to national recommendations and available vitamin $\mathrm{K}$ formulations. Methods Paediatricians reported cases of VKDB in infants aged $<6$ months and provided demographic, clinical and biochemical information via the Australian Paediatric Surveillance Unit.

Results 58 cases were reported, of which 5 (9\%) were early, 11 (19\%) classic and 42 (72\%) late VKDB. $53(91 \%)$ were exclusively breast fed. Seven (12\%) received oral prophylaxis, the majority (86\%) of whom did not receive all three recommended doses. The overall reported incidence was 0.84 per 100000 live births ( $95 \% \mathrm{Cl}: 0.64$ to 1.08 ) and the incidence of late VKDB was 0.61 per 100000 live births ( $95 \%$ Cl: 0.44 to 0.82 ), which are similar to rates reported by other countries where intramuscular vitamin $\mathrm{K}$ is recommended. VKDB rates were significantly higher (2.46 per 100000 live births; 95\% Cl: 1.06 to 4.85) between 1993 and March 1994 when oral prophylaxis was recommended $(p<0.05)$. Vitamin $\mathrm{K}$ was not given to $33(57 \%)$ cases, primarily due to parental refusal, and the number of parental refusals increased significantly after 2006 $(p<0.05)$. There were six deaths, all due to intracranial haemorrhage, and three associated with home delivery and parental refusal of vitamin $\mathrm{K}$.

Conclusions Incidence rates of VKDB in Australia are among the lowest in the world; however, we have identified an increasing trend of parental refusal. Ongoing surveillance and educational campaigns for health professionals and parents are needed to prevent VKDB.

\section{INTRODUCTION}

Vitamin K deficiency bleeding (VKDB) of infancy is a rare but potentially fatal disorder that affects 4.4-10.5 per 100000 live births, most of whom do not receive vitamin $\mathrm{K}$ prophylaxis at birth. ${ }^{1}$ Vitamin $\mathrm{K}$ is required for coagulation, but only small quantities cross the placenta and are found in breast milk so vitamin $\mathrm{K}$ prophylaxis is recommended routinely at birth to prevent VKDB. ${ }^{2}$ VKDB is classified according to the age of onset of bleeding: 'early' occurs within the first 24 hours of life; 'classic' between 1 and 7 days and 'late' presents between 1 week and 6 months of age, with intracranial haemorrhage in approximately $50 \%{ }^{3}$

Prophylaxis with intramuscular vitamin $\mathrm{K}$ was introduced in 1961. In 1992, an association between

\section{What is already known?}

Vitamin $\mathrm{K}$ deficiency bleeding (VKDB) is a rare but potentially fatal disorder which is almost entirely preventable by adequate vitamin $\mathrm{K}$ prophylaxis at birth.

- Intramuscular vitamin $\mathrm{K}$ is widely considered the gold standard and is the most reliable and efficient form of prophylaxis.

- Hepatobiliary disease is a recognised risk factor for prophylactic failure and early screening increases survival.

\section{What this study adds?}

- The incidence in VKDB decreased significantly after the national recommendations changed from oral to intramuscular vitamin $\mathrm{K}$ administration.

- Parental refusal of vitamin $\mathrm{K}$ has increased in the last decade, particularly in areas where immunisation refusal is most prevalent.

- Failure of intramuscular vitamin $\mathrm{K}$ prophylaxis in four late cases of VKDB in breastfed infants was reported and requires further research to identify risk factors.

childhood cancer and intramuscular vitamin $\mathrm{K}$ was reported. ${ }^{4}$ In response, in 1993, the National Health and Medical Research Council (NHMRC) of Australia released recommendations favouring oral prophylaxis. ${ }^{5}$ It was soon recognised that the evidence did not support an association between intramuscular vitamin $\mathrm{K}$ and childhood cancer. ${ }^{6}$ The revised NHMRC guidelines (1995) recommended intramuscular administration, with an option for three $1 \mathrm{mg}$ oral doses. ${ }^{7}$ Current (2010) NHMRC guidelines recommend $1 \mathrm{mg}$ intramuscular vitamin $\mathrm{K}$ at birth, with the alternative of two to three $2 \mathrm{mg}$ oral doses if parents refuse intramuscular therapy. ${ }^{8}$ Vitamin K in Australia is primarily administered via intramuscular injection. There are no national data collections on the administration of vitamin $\mathrm{K}$ prophylaxis and therefore national coverage is not known. Estimates from the largest jurisdiction in Australia, New South Wales (NSW), suggest that $96 \%$ of infants receive intramuscular vitamin K. ${ }^{9}$

In addition to changes in recommendations, there was a change in the vitamin $\mathrm{K}$ preparation available 
in Australia over the study period. The cremophore formulation (Konakion, $1 \mathrm{mg} / 0.5 \mathrm{~mL}$ Roche) which was licensed for intramuscular administration only (but was used for oral administration when parents refused consent for intramuscular) was replaced by Konakion MM Paediatric (Roche) in the year $2000 .^{8}$ This formulation continues to be the only vitamin $\mathrm{K}$ formulation available in Australia for prevention of VKDB. ${ }^{8}$ Konakion MM Paediatric contains $2 \mathrm{mg}$ in $0.2 \mathrm{~mL}$ vitamin $\mathrm{K} 1$ (phytomenadione) in a mixed-micellar formulation designed to afford better bio-availability, ${ }^{10}$ and is licensed for intramuscular and oral use. ${ }^{8}$

A single dose of $1 \mathrm{mg}$ intramuscular vitamin $\mathrm{K}$ is considered the gold standard because it consistently prevents most cases of late $\mathrm{VKDB}^{11}$ and is more effective than three $1 \mathrm{mg}$ or $2 \mathrm{mg}$ oral doses. ${ }^{12} 13$ It is considered the most reliable method of prophylaxis by the NHMRC because it eliminates risks associated with oral dosing regimens, including incomplete compliance and vitamin $\mathrm{K}$ malabsorption in babies with cholestasis. ${ }^{8}$

The Australian Paediatric Surveillance Unit (APSU) has collected information on cases of VKDB since 1993, providing unique data to estimate incidence during three policy changes and a change in the available vitamin $\mathrm{K}$ formulation.

\section{METHODS}

\section{Case definition}

Australian paediatricians and other child health professionals who participate in APSU surveillance were asked to report any infant aged $<6$ months with newly diagnosed VKDB. VKDB was defined as spontaneous bruising, bleeding or intracranial haemorrhage associated with prolonged clotting times but not due to disseminated intravascular coagulation or an inherited coagulopathy. VKDB was classified according to age of onset of bleeding: 'early' within 24 hours of birth; 'classic' between 1 and 7 days and 'late' between 1 week and 6 months of age. In addition, definite cases had to meet laboratory criteria: abnormal clotting time as indicated by international normalised ratio $\geq 4$ or prothrombin time (PT) $\geq$ four times the laboratory control value; normal or raised platelet count; normal fibrinogen or absence of fibrin degradation products; and evidence of normalisation of PT after vitamin $\mathrm{K}$ administration, when available. $^{14}$

\section{Reporting}

Like other paediatric surveillance units, the APSU facilitates prospective case reporting by paediatricians and other child health specialists. ${ }^{15-17}$ From 1993 to 2017 , APSU sent a monthly report card listing conditions of interest including VKDB to between 990 (in 1993) and 1500 (in 2017) paediatricians and over $90 \%$ of all cards were returned. For each case reported, clinicians provided information on maternal demographics, vitamin $\mathrm{K}$ prophylaxis, clinical presentation, feeding, coagulation studies and clinical outcome. Minimal identifiers (date of birth, and the first two letters of the infant's first and surnames) allowed identification of duplicate reports. Completed questionnaires were reviewed by the authors and an expert panel of clinicians, and cases that satisfied case definition criteria were classified as 'definite'. Cases with a history of bleeding consistent with VKDB but with incomplete coagulation results were classified as 'probable'. Cases with insufficient clinical or laboratory data to allow classification as definite or probable, and cases that did not satisfy the case criteria, were not included in analyses. Case criteria remained unchanged throughout the study.
Table 1 Characteristics of definite and probable cases, grouped by type, $\mathrm{n}(\%)$

\begin{tabular}{|c|c|c|c|c|}
\hline & Early & Classical & Late & Total \\
\hline $\mathrm{N}$, total & $5(9 \%)$ & $11(19 \%)$ & $42(72 \%)$ & $58(100 \%)$ \\
\hline $\mathrm{N}$, definite & 4 & 7 & 36 & 47 \\
\hline $\mathrm{N}$, probable & 1 & 4 & 6 & 11 \\
\hline Home birth & $2(40)$ & $0(0)$ & $11(26)$ & $13(22)$ \\
\hline \multicolumn{5}{|l|}{ Gestational age } \\
\hline Full term ( $\geq 37$ weeks) & $5(100)$ & $11(100)$ & $38(90)$ & $54(93)$ \\
\hline Premature (<37 weeks) & $0(0)$ & $0(0)$ & $3(7)$ & $3(5)$ \\
\hline Unknown & $0(0)$ & $0(0)$ & $1(2)$ & $1(2)$ \\
\hline \multicolumn{5}{|l|}{ Birth weight } \\
\hline Normal ( $\geq 2500 \mathrm{~g})$ & $5(100)$ & $9(82)$ & $25(60)$ & $39(67)$ \\
\hline Low $(<2500 \mathrm{~g})$ & $0(0)$ & $1(9)$ & $4(10)$ & $5(9)$ \\
\hline Unknown & $0(0)$ & $1(9)$ & $13(31)$ & $14(24)$ \\
\hline Vitamin K prophylaxis given & $2(40)$ & $5(45)$ & $18(43)$ & $25(43)$ \\
\hline Intramuscular & $1(20)$ & $1(9)$ & $12(29)$ & $14(24)$ \\
\hline Oral & $1(20)$ & $2(18)$ & $4(10)$ & $7(12)$ \\
\hline Intravenous & $0(0)$ & $1(9)$ & $0(0)$ & $1(2)$ \\
\hline Combination & $0(0)$ & $1(9)$ & $2(5)$ & $3(5)$ \\
\hline No vitamin K prophylaxis & $3(60)$ & $6(55)$ & $24(57)$ & $33(57)$ \\
\hline Parental refusal & $3(60)$ & $5(45)$ & $20(48)$ & $28(48)$ \\
\hline Not offered/error & $0(0)$ & $1(9)$ & $4(10)$ & $5(9)$ \\
\hline \multicolumn{5}{|l|}{ Feeding } \\
\hline Exclusively breast fed & $4(80)$ & $10(91)$ & $39(93)$ & $53(91)$ \\
\hline Predominately breast fed & $1(20)$ & $0(0)$ & $1(2)$ & $2(3)$ \\
\hline Predominately formula fed & $0(0)$ & $1(9)$ & $1(2)$ & $2(3)$ \\
\hline Unknown & $0(0)$ & $0(0)$ & $1(2)$ & $1(2)$ \\
\hline \multicolumn{5}{|l|}{ Site of bleeding } \\
\hline Skin bruising & $1(20)$ & $3(27)$ & $22(52)$ & $26(45)$ \\
\hline Umbilical & $2(40)$ & $1(9)$ & $3(7)$ & $6(10)$ \\
\hline Gastrointestinal & $2(40)$ & $7(64)$ & $11(26)$ & $20(34)$ \\
\hline Intracranial & $1(20)$ & $0(0)$ & $16(38)$ & $17(29)$ \\
\hline Nasal & $1(20)$ & $0(0)$ & $4(10)$ & $5(9)$ \\
\hline Heel prick ooze & $0(0)$ & $2(18)$ & $1(2)$ & $3(5)$ \\
\hline Circumcision site & $0(0)$ & $0(0)$ & $3(7)$ & $3(5)$ \\
\hline Jaundice in first week of life & $0(0)$ & $0(0)$ & $17(40)$ & $17(29)$ \\
\hline Liver disease confirmed & $0(0)$ & $0(0)$ & $18(43)$ & $18(31)$ \\
\hline Age of onset of VKDB, mean & $<1$ day & 2.4 days & 50.9 days & 37.1 days \\
\hline \multicolumn{5}{|l|}{ Outcome at discharge } \\
\hline No ongoing morbidity & $4(80)$ & $10(91)$ & $20(48)$ & $34(59)$ \\
\hline Ongoing morbidity & $0(0)$ & $1(9)$ & $14(33)$ & $15(26)$ \\
\hline Died & $0(0)$ & $0(0)$ & $6(14)$ & $6(10)$ \\
\hline Unknown & $1(20)$ & $0(0)$ & $2(5)$ & $3(5)$ \\
\hline
\end{tabular}

\section{Statistical analysis}

Incidence rates per 100000 live births and 95\% CIs were calculated using standard methods for rare events assuming a Poisson distribution. Estimates of live births, published by the Australian Bureau of Statistics, were used as the denominator. ${ }^{18}$ Two-tailed Fisher's exact test was used to compare incidence rates.

\section{RESULTS}

\section{Notifications}

There were 176 notifications between January 1993 and December 2017, for which 141 (80\%) had complete clinical data. In all, 38 were errors, 45 were duplicate reports and 58 were cases (47 definite and 11 probable). Two infants born in late 1992 who presented with VKDB in 1993 were included.

\section{Case summary}

Of the 58 cases, $42(72 \%)$ were classified as late VKDB (table 1), 17 of whom had jaundice prior to bleeding; 11 (19\%) were 


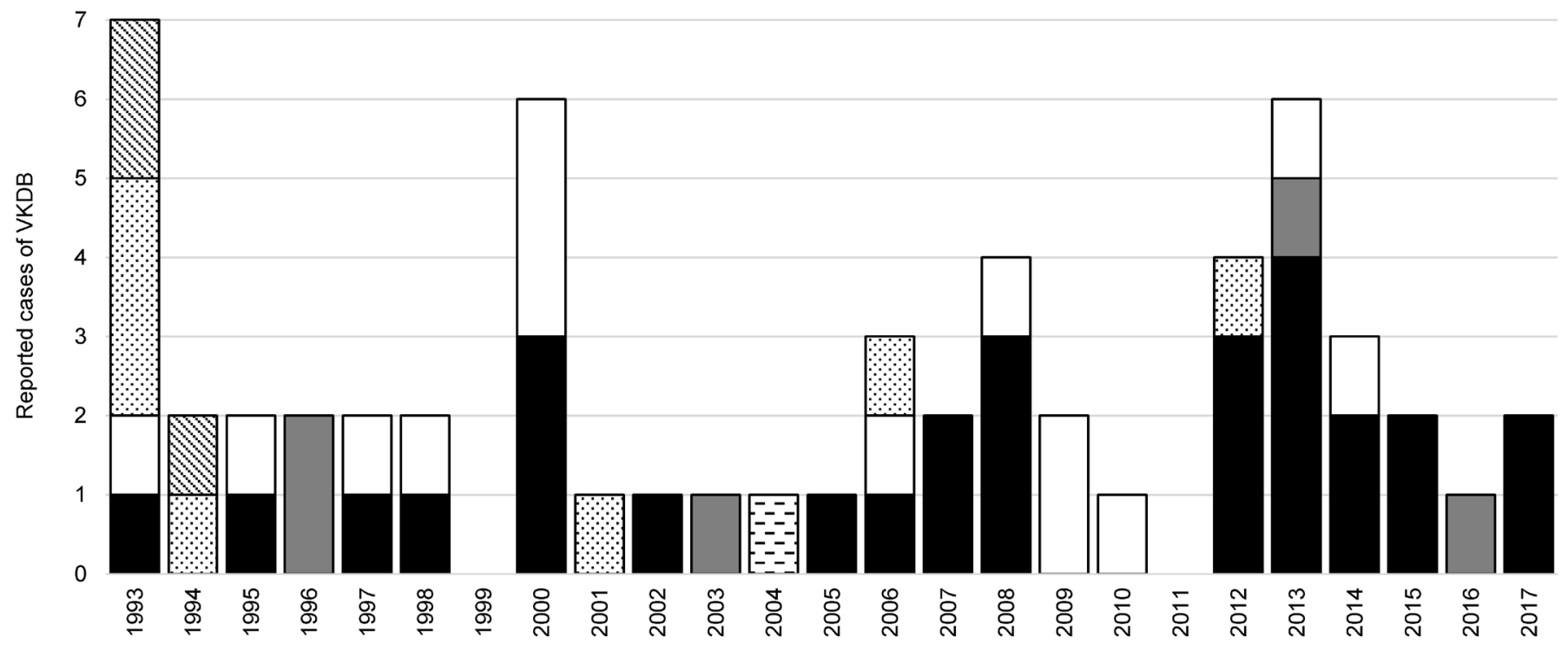

Figure 1 Reported cases of VKDB reported each year and grouped by the type of prophylaxis received or not received (1993-2017). 1. Not offered or omitted due to administrative errors. 2. Combined refers to two or more different routes of administration being used together (eg, oral and intramuscular, or intravenous and intramuscular, etc). VKDB, vitamin $\mathrm{K}$ deficiency bleeding.

classical VKDB and $5(9 \%)$ early VKDB. The majority were born at term (93\%) and exclusively breast fed (91\%). All three premature infants were exclusively breast fed: two of these have been previously described. ${ }^{19}$ In all, 13 (22\%) were delivered at home. The most common sites of bleeding were cutaneous (45\%), gastrointestinal (34\%) and intracranial (29\%).

Vitamin K was not administered to 33 (57\%) infants: consent was withheld for $28(85 \%)$ of these; in four (7\%), the reasons for not receiving vitamin $\mathrm{K}$ were not reported; and in one there was no clinician at the home birth to offer vitamin $\mathrm{K}$.

In all, 14 (24\%) infants bled after receiving intramuscular prophylaxis. Eight received the recommended $1 \mathrm{mg}$ dose, three received $0.1 \mathrm{mg}$ and three received an unknown dose. Of the eight who received the recommended $1 \mathrm{mg}$ dose, one was a classical case (bleeding at day 5) and the other 7 were late cases who bled between day 8 and 24 weeks of age. This is the only case of classic VKDB reported after receipt of $1 \mathrm{mg}$ intramuscular vitamin $\mathrm{K}$ in 25 years. The other seven were late cases who bled between day 8 and 24 weeks of age. Three of the late cases had liver or biliary disease and four were otherwise-healthy infants who were exclusively breast fed with no other recognised risk factors.

The three infants who received intramuscular vitamin $\mathrm{K} 0.1 \mathrm{mg}$ were born at term in 1993, 2008 and 2014. One had haematemesis on day 5 and was given one oral dose of $1 \mathrm{mg}$ vitamin $\mathrm{K}$ after which the coagulation studies were normal. The second infant had an intracranial bleed on day 98: vitamin $\mathrm{K}$ corrected the coagulation studies and biliary atresia was diagnosed. The third infant had skin bruising and bleeding which stopped after one dose of $1 \mathrm{mg}$ intramuscular vitamin K. Cases of VKDB following intramuscular prophylaxis were evenly distributed throughout the period of surveillance (figure 1).

Seven (12\%) infants developed VKDB following oral prophylaxis. One infant received three $1 \mathrm{mg}$ doses, two received two doses and four received one dose. All infants were exclusively $(83 \%)$ or predominately $(17 \%)$ breast fed and four of the five with late VKDB had confirmed liver disease. Almost half (43\%) the infants who received oral vitamin K were born between January 1993 and March 1994 and during this period the proportion of cases of VKDB following oral prophylaxis was significantly higher than after the introduction of the intramuscular recommendation in $1995\left(\chi^{2}=5.65\right.$; $\mathrm{p}=0.017$ ).

One infant received two doses of intravenous prophylaxis in 2004. Three infants received vitamin $\mathrm{K}$ by combined routes: two received intravenous and oral vitamin $\mathrm{K}$, and another infant received intramuscular and oral vitamin $\mathrm{K}$.

The proportion of parents who refused vitamin $\mathrm{K}$ was significantly higher during 2006-2017 (19, 63\%) compared with the period $1993-2005(9,32 \%)\left(\chi^{2}=5.64 ; p<0.05\right)$. In the 5 years 2013-2017, among the 14 cases of VKDB reported in this period, 10 (71\%) did not received vitamin $\mathrm{K}$ because their parents refused consent (figure 2). Parental refusal of vitamin K occurred at $11(85 \%)$ of the 13 home births. In total, $11(39 \%)$ cases following parental refusal occurred in Northern NSW and South-Eastern Queensland.

\section{Outcome}

Six deaths were reported, all from intracranial haemorrhage in infants with late VKDB. Three of these infants were delivered at home, were not given vitamin $\mathrm{K}$ due to parental refusal and were exclusively breast fed; one of whom had cholestasis. The remaining three were delivered in hospital and received intramuscular prophylaxis: two had confirmed liver disease and did not receive the recommended dose; the other did not have liver disease and had received recommended prophylaxis but was exclusively breast fed. One death occurred in 1993, 1998, 2010 and 2014 and two in 2000. No adverse effects following vitamin $\mathrm{K}$ administration were reported during the study period. 


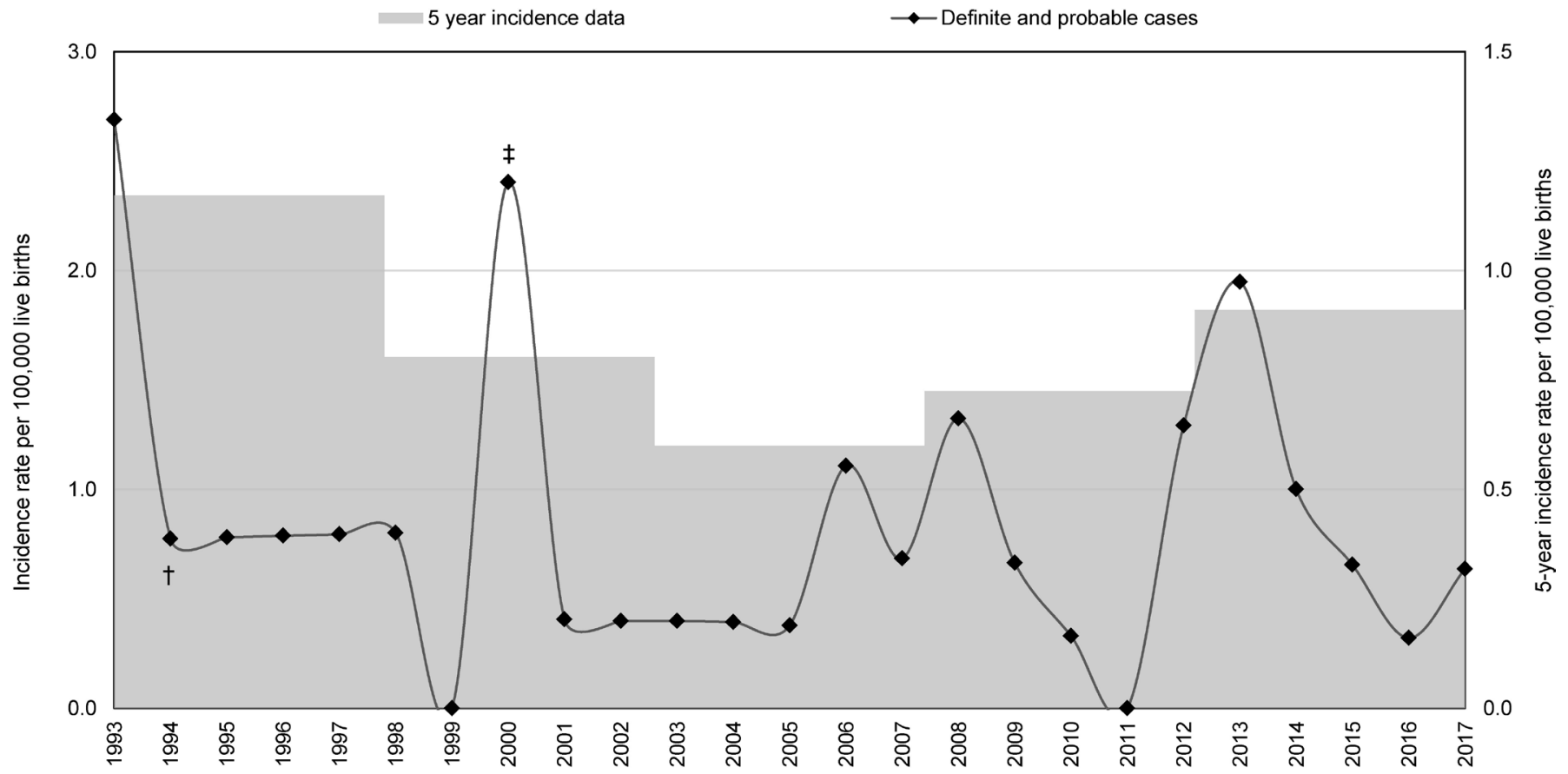

Figure 2 Estimated annual incidence rates and 5-year incidence rates of VKDB (1993-2017). †Before April 1994, oral vitamin K was recommended; from April 1994 intramuscular vitamin $\mathrm{K}$ was recommended. ¥The available formulation of vitamin $\mathrm{K}$ changed from Konakion, $1 \mathrm{mg} / 0.5 \mathrm{~mL}$ (Roche) to Konakion MM paediatric (Roche) and in the same year the National Health and Medical Research Council joint statement and recommendations on vitamin $\mathrm{K}$ administration to newborn infants to prevent VKDB in infancy, was released. VKDB, vitamin K deficiency bleeding.

\section{Incidence}

The overall annual incidence of VKDB was $0.84 / 100000$ live births (95\% CI: 0.64 to 1.08 ) and, for late VKDB, $0.61 / 100$ 000 live births $(95 \% \mathrm{CI}: 0.44$ to 0.82$)$. Incidence rates peaked in 1993, 2000 and 2013 (figure 2). Between January 1993 and March 1994, when oral vitamin K was recommended, the incidence per 100000 live births was significantly higher than during the remainder of the study when intramuscular prophylaxis was recommended, both overall $(2.46,95 \% \mathrm{CI}: 1.06$ to 4.85 vs $0.76,95 \% \mathrm{CI}$ : 0.56 to $1.00 ; \mathrm{p}=0.011)$ and for late VKDB $(1.85$, $95 \% \mathrm{CI}$ : 0.68 to 4.02 vs 0.5 , $95 \% \mathrm{CI}$ : 0.38 to 0.76 ; $\mathrm{p}=0.026$ ).

\section{DISCUSSION}

To our knowledge, we report the longest period of continuous national surveillance for VKDB, which covered changes in policy and vitamin $\mathrm{K}$ formulation in Australia. The surveillance method used is similar to other countries. ${ }^{16}$ The overall annual incidence of VKDB in Australia, at 0.84 per 100000 live births, is comparable to rates reported in countries where $1 \mathrm{mg}$ intramuscular or the three $2 \mathrm{mg}$ oral dosing regimens were used, although international comparison is limited by slightly different surveillance methods and timing of studies. ${ }^{12} 1320-22$

VKDB incidence decreased significantly after the Australian recommendations were changed from three $1 \mathrm{mg}$ doses of oral vitamin $\mathrm{K}$ to $1 \mathrm{mg}$ intramuscular injection in April 1994. The 1994 recommendations retained an option for parents to choose the oral regimen and several cases of VKDB were associated with oral dosing, mostly when fewer than three doses were given. In 2000 , the alternative oral regimen was changed from three $1 \mathrm{mg}$ to three $2 \mathrm{mg}$ doses and only three cases of VKDB following oral prophylaxis have been reported in the 17 years since.

Cases of VKDB were reported almost every year of the study. Incidence rates increased in the year 2000, coinciding with a change in the vitamin $\mathrm{K}$ preparation available in Australia from the Cremophor EL formulation (Konakion, Roche) for intramuscular injection to the mixed-micellar Konakion MM Paediatric (Roche) for either intramuscular or oral dosing. ${ }^{8}$ The increased incidence is unlikely to be related to the introduction of the mixed-micellar vitamin $\mathrm{K}$ formulation, which has not been associated with increasing rates of VKDB elsewhere. ${ }^{23}$ A possible explanation may be increased awareness and reporting of VKDB with the release of the new NHMRC guidelines on vitamin $\mathrm{K}$ prophylaxis in $2000 .^{8}$

According to our data, a single $1 \mathrm{mg}$ intramuscular dose of vitamin $\mathrm{K}$ at birth continues to prevent almost all cases of late VKDB. The higher incidence rates of late VKDB compared with other countries with similar prophylactic regimens might be explained by the increase in parental refusals in Australia; only New Zealand reported a higher incidence of late VDKB which was almost exclusively attributed to parental refusal of vitamin $\mathrm{K}^{22}$ The number of VKDB cases following parental refusals doubled in the second half of the study and further increased in the last 5 years. Increasing rates of parental refusal have been reported worldwide, ${ }^{24}$ and Busfield reported parental refusal in 45\% of VKDB cases between 2006 and 2008 in the $\mathrm{UK}^{20}$ In NSW, the most populous jurisdiction of Australia, it is estimated that approximately $1.1 \%$ of babies do not receive vitamin $\mathrm{K}$ prophylaxis because of parental refusal. ${ }^{9}$ Analysis of the geographical distribution of the infants with VKDB whose parents refused vitamin $\mathrm{K}$ showed a cluster in Northern NSW and South East Queensland, areas where immunisation refusal rates are highest in Australia. ${ }^{26}$ Correlation between refusal of vitamin $\mathrm{K}$ and refusal of routine vaccinations has been previously reported. ${ }^{27}$ The reasons for parental refusal are manifold: vitamin $\mathrm{K}$ is considered unnatural, potentially painful, incongruous with an 'alternative' lifestyle and concerns about the increased risk of cancer persist. ${ }^{28}$ In New Zealand, midwives were half as likely as doctors to support vitamin $\mathrm{K}$ prophylaxis. ${ }^{29}$ Home births are 
associated with higher rates of refusal of vitamin $K,{ }^{30}$ and home births accounted for over a third (39\%) of parental refusals while only representing $0.3 \%$ of all Australian births. ${ }^{31}$

There were four infants with VKDB in our study who were born in hospital and did not receive vitamin $\mathrm{K}$ despite parental consent. Furthermore, three infants with VKDB received a lower than recommended dose of $0.1 \mathrm{mg}$ intramuscular instead of $1 \mathrm{mg}$ intramuscularly. These errors are rare but highlight the need for verification protocols to ensure neonates are not inadvertently overlooked or given the wrong dose. Education and awareness campaigns for parents and for health professionals who attend births are needed. Strategies requiring the involvement of senior paediatricians in all cases where parents refuse vitamin $\mathrm{K}$ have been suggested in the UK and could be implemented in Australia. ${ }^{32}$

Failure of vitamin $\mathrm{K}$ prophylaxis is rare, with an estimated incidence rate of $0-0.25$ per 100000 live births. ${ }^{33}$ Of the seven cases of late VDKB following $1 \mathrm{mg}$ intramuscular vitamin $\mathrm{K}$, three had confirmed liver or biliary disease, which is associated with prophylactic failure, ${ }^{11}$ but four exclusively breastfed infants without other risk factors had VKDB after $1 \mathrm{mg}$ intramuscular vitamin K prophylaxis. Prophylaxis failure has been reported in healthy breastfed infants. ${ }^{21}{ }^{34}$ Exclusive breastfeeding is widely recommended until 6 months of age, ${ }^{35}$ so the relationship between breastfeeding, mothers' nutritional status and VKDB in infants who received intramuscular vitamin $\mathrm{K}$ at birth warrants further investigation.

There was one report of classic VKDB following prophylaxis with the recommended $1 \mathrm{mg}$ intramuscular dose. The data available to the APSU are not extensive enough to further investigate in detail the cause of VKDB in this rare case, and failure of prophylaxis cannot be confirmed. It is more plausible that the apparent failure of intramuscular prophylaxis in one classic case reported by us and another case reported by the British Paediatric Surveillance Unit (BPSU) ${ }^{36}$ is instead a failure of administration of vitamin $\mathrm{K}$ or a reporting error by the clinicians. Comparison of the number of cases of VKDB after apparent administration of $1 \mathrm{mg}$ intramuscular vitamin $\mathrm{K}$ between the BPSU and APSU is difficult because the APSU data represent continuous surveillance over 25 years (1993-2017) while the four BPSU studies covered several disjointed periods: 1988-1990, 1993-1994, 2001-2002 and 2006-2008. ${ }^{2036} 37$ Furthermore, as both the APSU and BPSU rely on voluntary reporting by clinicians, there is possible over-reporting or under-reporting in either system.

Since the return to the recommendation for intramuscular prophylaxis in 1994, there have been four cases of VKDB after incomplete oral dosing. Lack of compliance with three oral doses is recognised as a potential problem in Australia ${ }^{38}$ and elsewhere, ${ }^{39}$ but has been dismissed by others. ${ }^{40}$ Intravenous vitamin $\mathrm{K}$ prophylaxis does not provide the lasting protection achieved with intramuscular vitamin $\mathrm{K}^{19}$ and has never been recommended in Australia, although it was given alone to one infant in 2004, and in combination with other modes of administration to three children between 1993 and 1994.

\section{LIMITATIONS AND STRENGTHS}

The APSU relies on reporting by clinicians and there is likely case under-ascertainment in our study. The reported incidence rates must be considered as minimum estimates only. In all, 35 cases could not be classified because of incomplete data. As most of these cases were from 1993, the incidence may be significantly under-estimated for that year. Testing for protein induced by vitamin $\mathrm{K}$ absence/antagonism of clotting factor II (PIVKA-II; undercarboxylated prothrombin) is not routinely used in Australia and no information was collected on this measure during our study. This should be considered for future surveillance protocols, especially if PIVKA-II testing becomes more common. ${ }^{32}$ Despite these limitations, we achieved a high monthly return rate of report cards (93.5\%) suggesting a high level of participation among clinicians enrolled in the APSU. ${ }^{15}$

\section{CONCLUSION}

Parents continue to withhold consent for vitamin $\mathrm{K}$ prophylaxis and VKDB following refusal has increased in the last 5 years. Research about community perceptions regarding the importance of vitamin $\mathrm{K}$ prophylaxis and education is needed. Clinicians should be aware of the risk of VKDB in infants with liver or biliary disease. Education about national guidelines is needed for health professionals involved in perinatal care and for the community regarding the importance of vitamin $\mathrm{K}$ prophylaxis and potential consequences of vitamin $\mathrm{K}$ refusal.

\section{Author affiliations}

${ }^{1}$ Australian Paediatric Surveillance Unit, The University of Sydney, Sydney, New South Wales, Australia

${ }^{2}$ Australian Institute of Health Innovation, Partnership Centre in Health System Sustainability, Macquarie University, Sydney, New South Wales, Australia

${ }^{3}$ Faculty of Medicine and Health, University of Sydney, Sydney, New South Wales, Australia

${ }^{4}$ Population Health Intelligence, Healthy People and Places Unit, South Western Sydney Local Health District, Liverpool, New South Wales, Australia

${ }_{5}^{5}$ School of Public Health and Community Medicine, University of New South Wales, Sydney, New South Wales, Australia

${ }^{6}$ Faculty of Medicine and Health, Discipline of Child and Adolescent Health, University of Sydney, Sydney, New South Wales, Australia

${ }^{7}$ Kids Research, Sydney Children's Hospitals Network Randwick and Westmead, Sydney, New South Wales, Australia

Acknowledgements We thank all paediatricians and other child health clinicians who have participated in APSU surveillance activities since 1993 and contributed information about cases of VKDB. We acknowledge funding support from the Australian Department of Health, in-kind support from the Royal Australasian College of Physicians, Division of Paediatrics and Child Health; Kids Research at the Sydney Children's Hospital at Westmead and The University of Sydney, Faculty of Medicine and Health, Discipline of Child and Adolescent Health. We thank Professor Henderson-Smart who was the driving force in establishing surveillance for VKDB in Australia, and Dr Peter Loughnan, Dr Kerry Chant, Dr Greta Ridley and Dr Lee Taylor for their assistance with the surveillance study. We thank Ms Amy Phu who developed an electronic data capture form in REDCap for use from 2013 onwards. This surveillance study was sponsored by Roche Pty Ltd between the years 2000-2005. YZ was supported in-part by a Sydney Medical School Foundation Fellowship; EE was supported by NHMRC Practitioner Fellowships (\# 457084, 1021480 and 1135959).

Contributors EE and BJ developed the original study protocol and have been involved in collection, analysis and interpretation of data since 1993. CG, YZ and BJ analysed and interpreted data and drafted the manuscript which has had input from all authors. $C G$ and $Y Z$ are joint first authors.

Competing interests None declared.

\section{Patient consent for publication Not required.}

Ethics approval The study received approval from the Sydney Children's Hospitals Network Human Research Ethics Committee (Approval number: 2006-062).

Provenance and peer review Not commissioned; externally peer reviewed.

Data availability statement Data are available upon reasonable request.

ORCID iD

Cameron J Grover http://orcid.org/0000-0002-4866-4805

\section{REFERENCES}

1 von Kries R, Hanawa Y. Neonatal vitamin K prophylaxis. Report of scientific and standardization Subcommittee on perinatal haemostasis. Thromb Haemost 1993;69:293-5.

2 World Health Organization. Recommendations for management of common childhood conditions: evidence for technical update of pocket book recommendations: newborn conditions, dysentery, pneumonia, oxygen use and delivery, common causes of fever, 
severe acute malnutrition and supportive care. Geneva: World Health Organization, 2012. https://apps.who.int/iris/bitstream/handle/10665/44774/9789241502825_eng. pdf. (Accessed January 2018).

3 Loughnan PM, McDougall PN. Epidemiology of late onset haemorrhagic disease: a pooled data analysis. J Paediatr Child Health 1993;29:177-81.

4 Golding J, Greenwood R, Birmingham K, et al. Childhood cancer, intramuscular vitamin K, and pethidine given during labour. BMJ 1992;305:341-6.

5 National Health and Medical Research Council. Joint statement and interim recommendations on vitamin $\mathrm{K}$ prophylaxis for haemorrhagic disease in infancy. J Paediatr Child Health 1993;29:182.

6 Roman E, Fear NT, Ansell P, et al. Vitamin K and childhood cancer: analysis of individual patient data from six case-control studies. Br J Cancer 2002;86:63-9.

7 National Health and Medical Research Council. Vitamin K prophylaxis for haemorrhagic disease of the newborn : advice for health care workers. Canberra: Australian Govt. Pub. Service, 1995.

8 National Health and Medical Research Council. Joint statement and recommendations on vitamin $\mathrm{K}$ administration to newborn infants to prevent vitamin K deficiency bleeding in infancy. Canberra: National health and medical Research Council, 2010. Available: https://www.nhmrc.gov.au/about-us/ publications/vitamin-k-administration-newborns-joint-statement\#block-viewsblock-file-attachments-content-block-1 [Accessed January 2018].

9 Khambalia AZ, Roberts CL, Bowen JR, et al. Maternal and infant characteristics by mode of vitamin $\mathrm{K}$ prophylaxis administration. J Paediatr Child Health 2012;48:665-8

10 Amédée-Manesme O, Lambert WE, Alagille D, et al. Pharmacokinetics and safety of a new solution of vitamin K1(20) in children with cholestasis. J Pediatr Gastroenterol Nutr 1992;14:160-5.

11 Shearer MJ. Vitamin K deficiency bleeding (VKDB) in early infancy. Blood Rev 2009;23:49-59.

12 von Kries R, Hachmeister A, Göbel U. Can 3 oral 2 mg doses of vitamin K effectively prevent late vitamin K deficiency bleeding? Eur J Pediatr 1999;158 Suppl 3:S183-S186

13 Laubscher B, Bänziger O, Schubiger G, et al. Prevention of vitamin K deficiency bleeding with three oral mixed micellar phylloquinone doses: results of a 6 -year (2005-2011) surveillance in Switzerland. Eur J Pediatr 2013;172:357-60.

14 Tripp JH, Cornelissen EAM, Loughnan P, et al. Suggested protocol for the reporting of prospective studies of vitamin K deficiency bleeding. In: Sutor AH, Hathaway WE, eds. Vitamin K in infancy. Stuttgart, Germany: Schattauer, 1995: 395-9.

15 He S, Zurynski YA, Elliott EJ. Evaluation of a national resource to identify and study rare diseases: the Australian paediatric surveillance unit. J Paediatr Child Health 2009:45:498-504.

16 Zurynski YA, Grenier D, Lynne R. International network of paediatric surveillance units: 15 years of international research into rare childhood diseases. INoPSU, 2013. Available: http://www.apsu.org.au/assets/INoPSU/15-year-report.pdf [Accessed January 2018].

17 Cornelissen $\mathrm{M}$, von Kries $\mathrm{R}$, Loughnan $\mathrm{P}$, et al. Prevention of vitamin $\mathrm{K}$ deficiency bleeding: efficacy of different multiple oral dose schedules of vitamin K. Eur J Pediatr 1997;156:126-30.

18 Australian Bureau of Statistics, ABS.Stat Beta. births, summary, by state. Available: http://stat.data.abs.gov.au/Index.aspx?DatasetCode=births_summary [Accessed June 2018].

19 Loughnan PM, McDougall PN, Balvin H, et al. Late onset haemorrhagic disease in premature infants who received intravenous vitamin K1. J Paediatr Child Health $1996 ; 32: 268-9$
20 Busfield A, Samuel R, McNinch A, et al. Vitamin K deficiency bleeding after NICE guidance and withdrawal of Konakion neonatal: British paediatric surveillance unit study, 2006-2008. Arch Dis Child 2013;98:41-7.

21 McMillan DD, Grenier D, Medaglia A. Canadian paediatric surveillance program confirms low incidence of hemorrhagic disease of the newborn in Canada. Paediatr Child Health 2004;9:235-8.

22 Darlow BA, Phillips AA, Dickson NP. New Zealand surveillance of neonatal vitamin $\mathrm{K}$ deficiency bleeding (VKDB): 1998-2008. J Paediatr Child Health 2011:47:460-4.

23 Schubiger G, Berger TM, Weber R, et al. Prevention of vitamin $\mathrm{K}$ deficiency bleeding with oral mixed micellar phylloquinone: results of a 6 -year surveillance in Switzerland. Eur J Pediatr 2003;162:885-8.

24 Schulte R, Jordan LC, Morad A, et al. Rise in late onset vitamin $\mathrm{K}$ deficiency bleeding in young infants because of omission or refusal of prophylaxis at birth. Pediatr Neurol 2014; 50:564-8.

25 Sahni V, Lai FY, MacDonald SE. Neonatal vitamin K refusal and nonimmunization. Pediatrics 2014;134:497-503.

26 National Centre for Immunisation Research and Surveillance. Annual immunisation coverage report, 2015. Available: http://www.ncirs.edu.au/assets/surveillance/ coverage/Annual-Immunisation-Coverage-Report-2015.pdf [Accessed February 2018]

27 Bernhardt H, Barker D, Reith DM, et al. Declining newborn intramuscular vitamin K prophylaxis predicts subsequent immunisation refusal: a retrospective cohort study. J Paediatr Child Health 2015;51:889-94.

28 Miller $\mathrm{H}$, Kerruish N, Broadbent RS, et al. Why do parents decline newborn intramuscular vitamin K prophylaxis? J Med Ethics 2016:42:643-8.

29 Gosai S, Broadbent RS, Barker DP, et al. Medical and midwifery attitudes towards vitamin K prophylaxis in New Zealand neonates. J Paediatr Child Health 2014; 50:536-9.

30 Hamrick HJ, Gable EK, Freeman EH, et al. Reasons for refusal of newborn vitamin K prophylaxis: implications for management and education. Hosp Pediatr 2016:6:15-21.

31 Australian Institute of Health and Welfare. Australia's Mothers and Babies 2015 - in brief. Canberra: Australian Institute of Health and Welfare, 2017. Available: https:// www.aihw.gov.au/reports/mothers-babies/australias-mothers-babies-2015-in-brief/ contents/table-of-contents [Accessed February 2018].

32 Clarke P, Shearer MJ. Vitamin K deficiency bleeding: the readiness is all. Arch Dis Child 2007;92:741-3.

33 von Kries R. Vitamin K prophylaxis--a useful public health measure? Paediatr Perinat Epidemiol 1992;6:7-13.

34 Martic J, Pejic K, Veljkovic D, et al. Late vitamin K deficiency bleeding despite intramuscular prophylaxis at birth - is there a need for additional supplementation? Srp Arh Celok Lek 2017:145:254-8.

35 Kramer MS, Kakuma R. Optimal duration of exclusive breastfeeding. Cochrane Database Syst Rev 2012;(8):CD003517.

36 McNinch A, Busfield A, Tripp J. Vitamin K deficiency bleeding in Great Britain and Ireland: British paediatric surveillance unit surveys, 1993-94 and 2001-02. Arch Dis Child 2007:92:759-66.

37 McNinch AW, Tripp JH. Haemorrhagic disease of the newborn in the British Isles: two year prospective study. BMJ 1991;303:1105-9.

38 Carmichael A. Vitamin K administration to the newborn: a public health policy and service delivery dilemma. J Paediatr Child Health 1993;29:167-8.

39 Doran O, Austin NC, Taylor BJ. Vitamin K administration in neonates: survey of compliance with recommended practices in the dunedin area. N Z Med J 1995; 108:337-9.

40 Loughnan PM, McDougall PN. The efficacy of oral vitamin K1: implications for future prophylaxis to prevent haemorrhagic disease of the newborn. J Paediatr Child Health 1993;29:171-6. 